\title{
Soft-Threshold-Based Multilayer Decision Feedback Equalizer (STM-DFE) Algorithm and VLSI Architecture
}

\author{
Chih-Hsiu Lin and An-Yeu (Andy) Wu
}

\begin{abstract}
The decision feedback equalizer (DFE) is an efficient scheme to suppress intersymbol interference (ISI) in various communication and magnetic recording systems. However, most cost-effective DFE implementations suffer from the phenomenon of error propagation, which degrades its bit error rate (BER) performance. This paper proposes a soft-threshold-based multilayer DFE (STM-DFE) technique to reduce the BER. It involves very low hardware overhead costs as compared to the conventional DFE. When applied to a practical Lorentzian channel and channels of different eigenvalue spread, the STM algorithm even outperforms the Ideal DFE (IDFE) system (in the IDFE, symbols are correctly fed back without propagation errors). Simulation results show that the proposed scheme can efficiently reduce the burst error length (BEL) as well as BER. Additionally, the hardware overhead to implement the STM-DFE algorithm is negligible compared with the conventional DFE. By using the concepts of Shanbhag and Parhi and of Yang, Wu, and Lai, we also propose the pipelined STM-DFE (PSTM-DFE) architecture for high-speed communication/storage applications.
\end{abstract}

Index Terms-DFE, multilayer, soft-threshold, STM-DFE.

\section{INTRODUCTION}

D ATA transmission through the band-limited channel suffers from time-domain dispersion, which causes intersymbol interference (ISI) and degrades the throughput rate [1]. The maximum likelihood sequence estimation (MLSE) is the optimal solution to solve the ISI problem. However, in most cases it reduces the bit error rate (BER) at the price of very high hardware complexity, especially for a large constellation of modulation and a large delay spectrum of a channel. To simplify this hardware complexity, the authors of [3] combined the MLSE with the decision feedback equalizer (DFE), in which the trellis states are partitioned to smaller subsets. This partition scheme can reduce the overall number of trellis states. However, it involves much higher hardware costs than the linear equalizer (LE) and conventional DFE.

Manuscript received January 16, 2004; revised September 30, 2004. This work was supported in part by MediaTek Incorporation and the National Science Council, R.O.C., under Grant NSC 90-2218-E-002-040. The associate editor coordinating the review of this manuscript and approving it for publication was Prof. Chaitali Chakrabarti.

C.-H. Lin is with the Graduate Institute of Electronics Engineering and Department of Electrical Engineering and the Access Lab., National Taiwan University, Taipei, 106 Taiwan, R.O.C. (e-mail: fil@access.ee.ntu.edu.tw).

A.-Y. (Andy) Wu is with the Graduate Institute of Electronics Engineering and Department of Electrical Engineering, National Taiwan University, Taipei, 106 Taiwan, R.O.C. (e-mail: andywu@ cc.ee.ntu.edu.tw).

Digital Object Identifier 10.1109/TSP.2005.851170

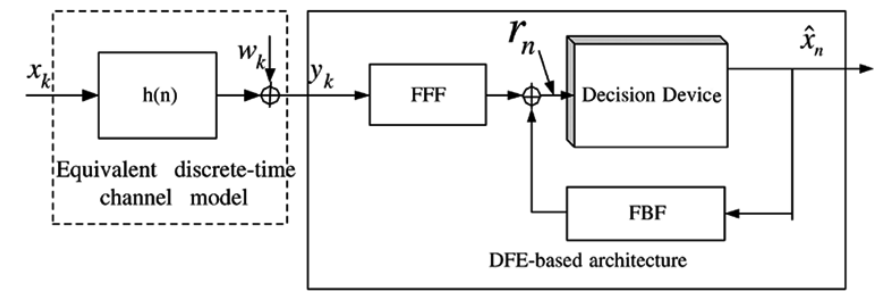

Fig. 1. Discrete-time channel model and the block diagram of the DFE-based design, where FBF and FFF denote the feed backward filter and feed forward filter, respectively.

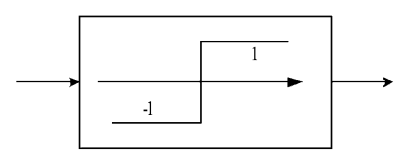

(a)

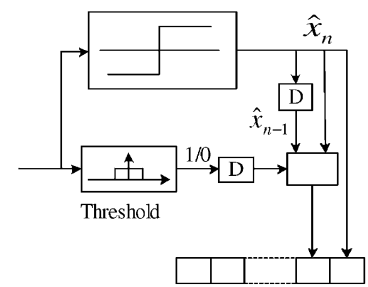

(c)

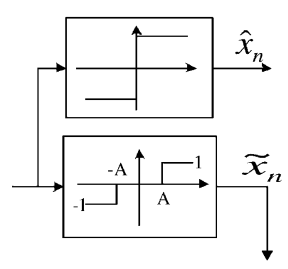

(b)

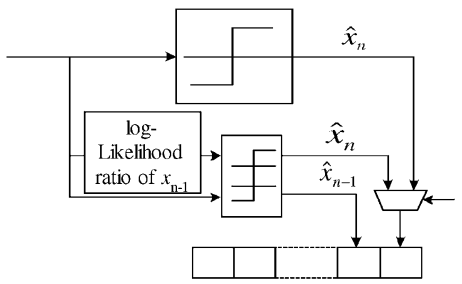

(d)
Fig. 2. Decision devices in DFE-based implementations. (a) Conventional slicer. (b) Modified slicer with erasure scheme [23]. (c) Threshold technique [6]. (d) Proposed STM-engine.

In general, the DFE has better performance than the LE. Hence, it is widely employed in practical designs. One of the major drawbacks of the DFE is error propagation, which is caused by slicing errors. Much research has focused on analyzing error propagation [20]-[22]. Some approaches suggest modification of the decision devices to reduce the error propagation [6], [23]. The right-hand side of Fig. 1 shows the generalized DFE architecture. Fig. 2(b)-(d) shows these various types of decision devices that aim at the reduction of error propagation in DFE-based designs. Fig. 2(a) shows the conventional DFE scheme, in which the decision device is just a slicer. Fig. 2(b) shows the erasure scheme [23]. In the erasure scheme, if the detected signal is smaller than a given threshold value, 0 is fed back; otherwise, it works as a slicer. In [5] and 
[6], the authors proposed a threshold technique (TT) approach to detect the error events. The idea is that the past decision errors that reside in the feedback register tend to cause an offset at the slicer. We call this scheme the threshold technique decision feedback equalizer (TT-DFE). The TT-DFE makes use of this offset and employs an offset-threshold slicer to detect the presence of error propagation. When the offset events are detected, they may be corrected in the next symbol. Fig. 2(c) illustrates the TT-DFE scheme. Additionally, a fast simulation procedure in [6] is also derived to estimate the BER. One of the disadvantages of the TT-DFE is that design parameters in the TT-DFE are difficult to determine, and they depend on the channel model. The performance will be degraded due to the nonoptimal design parameters. Hence, it limits the application of the TT-DFE to channels that are stationary and known in advance.

Inspired by the TT-DFE [6], we propose the soft-thresholdbased multilayer decision feedback equalizer (STM-DFE) to enhance the DFE by modifying the decision device. The key idea is to define a reliable/unreliable region. When the received signal is in the region, the STM-DFE does not make the decision instantly. Instead, it forwards the log-likelihood ratio to the next symbol. The process can be performed continuously until the output of equalizers becomes reliable or the last stage is reached. Then, the decision on the transmitted data is made at the same time. Fig. 2(d) illustrates the block diagram of the STM engine. In this paper, the threshold value of reliable/unreliable regions is analyzed in closed form. The STM algorithm can adjust the threshold value dynamically, which makes full use of the information from the output of the equalizers. It does not just detect it by a simple threshold device. The simulation results show that the BER and BEL of the proposed STM algorithm are better than those of the TT-DFE [6] and the conventional DFE when being applied to a Lorentzian channel [7], as well as in different eigenvalue spread channels [8]. Compared with the ideal decision feedback equalizer (IDFE), the STM-algorithm also has better performance.

In VLSI implementations, the least mean squared (LMS) algorithm is a well-known technique to update the weight coefficients of ADFE in a cost-efficient way. We apply the LMS algorithm to update the weight coefficient of the STM-DFE. However, the fine-grain pipelining of the ADFE is a difficult problem for high-speed applications due to the decision feedback loop (DFL). Similarly, it is also difficult to pipeline the STM-DFE VLSI architecture. The relaxed ADFE techniques [10] and predicted parallel branch slicer scheme (PPBS-ADFE) [13] solve the aforementioned problem with speed limitation. In this work, we also suggest applying the relaxed ADFE [10], [13] to derive the pipelined STM-DFE (PSTM-DFE) architecture. Thus, the speed performance can be enhanced.

The rest of the paper is organized as follows. Section II describes the channel model and reviews the recent work of [6]. In Section III, we present the two-layer STM algorithm and extend it to a higher order layer. The numerical analysis and simulation results are discussed in Section IV. The simplified architecture of the STM-DFE is given in Section V. The pipelined architecture is also proposed for high-speed applications. Finally, we conclude our work in Section VI.

\section{REVIEW OF THE TT-DFE APPROACHES [6]}

In this section, we review the recent work of the TT-DFE [6], which is a simple to reduce the error propagation of a DFE. The communication system adopted in this paper is shown in Fig. 1, in which the notations are defined as follows:

- $x_{k}$ is the transmitted data. In general, $x_{k}$ are assumed to be independent with zero-mean. $\hat{x}_{n}$ is the detection of $x_{n}$.

- $h(n)$ is the equivalent discrete-time channel impulse response, which is linear and bandlimited and shown on the left-hand side of Fig. 1.

- $w_{n}$ is Additive White Gaussian Noise (AWGN).

- $y_{n}$ is the channel output. It is considered to be corrupted by $w_{n}$ at the front end of the receivers.

- $r_{n}$ is the output of the equalizers and can be expressed as

$$
r_{n}=\sum_{m=0}^{N_{b}-1} b_{m} \times y_{n-m}-\sum_{m=1}^{N_{a}} a_{m} \times \hat{x}_{n-m}
$$

where

- $a_{k}$ denotes the $k$ th tap-weight of the feed backward filters (FBFs).

- $b_{k}$ denotes the $(k+1)$ th tap-weight of feed forward filters (FFFs).

- $N_{a}$ is the number of taps in the FBF.

- $N_{b}$ is the number of taps in the FFF.

In most DFE implementations, the receiver must determine the value of data instantly and then feed back the tentative decision. When the decision is wrong, the error will propagate throughout the feedback tap delay line. Moreover, it will affect the successive symbols to wrong decisions. The idea of the TT-DFE is as follows. When decision error arises at time $n$, it will cause a large offset the next time $(n+1)$. By detecting the large offset, the error event at time $n$ can be detected and recovered. We reformulate the TT-DFE algorithm as follows [6]:

$$
\begin{aligned}
r_{n} & =f_{0} x_{n}+\varepsilon_{n}^{d} & & \\
\text { Type 1: } r_{n+1} & =\left(f_{0}-2 a_{1}\right) x_{n+1}+\varepsilon_{n+1}^{d}, & & \text { for } x_{n+1}=x_{n} \\
\text { Type 2 }: r_{n+1} & =\left(f_{0}+2 a_{1}\right) x_{n+1}+\varepsilon_{n+1}^{d}, & & \text { for } x_{n+1}=x_{n}
\end{aligned}
$$

where

- $f_{0}$ is one tap-weight of FFF (it, in general, approximates to 1 );

- $\varepsilon_{n}^{d}$ is the noise, caused by the AWGN, error propagation, and nonperfect equalized weight coefficients.

Assuming that $2 a_{1}$ is not small relative to $f_{0}$, then $r_{n+1}$ will have a large offset to $x_{n+1}$ due to the decision error of $x_{n}$. The authors propose a threshold test to detect these types of decision errors at time $n$, when $r_{n}, r_{n+1}, x_{n}$, and $x_{n+1}$ satisfy

or

$$
\text { Type 1: }\left\{\begin{array}{l}
\left|r_{n}\right|<\alpha_{1} \\
\left|r_{n+1}\right|>\beta_{1} \\
\widehat{x}_{n+1} \neq \hat{x}_{n}
\end{array}\right.
$$

$$
\text { Type 2: }\left\{\begin{array}{l}
\left|r_{n}\right|<\alpha_{2} \\
\left|r_{n+1}\right|<\beta_{2} \\
\hat{x}_{n+1}=\hat{x}_{n}
\end{array}\right.
$$


where $\alpha_{1}, \alpha_{2}, \beta_{1}$, and $\beta_{2}$ are design parameters. The TT-DFE is very suitable for low-cost VLSI designs. The hardware overhead is just two threshold detectors. The drawback of this scheme is that the design parameters are difficult to determine, and these values are different case by case, especially when $a_{1}$ is not around \pm 0.5 .

\section{Proposed STM Algorithm}

To derive the STM-algorithm, three assumptions are made in this paper.

A1) The transmitted data sequence is independent with zero-mean.

A2) The noise $\varepsilon_{n}^{d} \equiv x_{n}-r_{n}$ is AWGN with zero-mean and variance $\sigma_{n}^{2}$. This assumption is commonly used in many mathematical derivations of common systems [24]-[28].

A3) For the convenience of our discussions, the detections of the transmitted data prior to $x_{n-k}$ are assumed to be correct when we want to make the decisions of these transmitted data from time $n$ to time $n-k$.

\section{A. Two-Layer STM Algorithm}

In this section, we first derive the two-layer STM algorithm. Then, we extend it to a higher order layer. For clarification of the representation, we assume that the date sequence is $\{-1,+1\}$. It is trivial to extend the data for higher order constellations. Let $r_{n}=x_{n}+\varepsilon_{n}^{d}$ [applying Assumption (A2)] at time $n$; the $\log$ likelihood ratio (LLR) of $x_{n}=-1$ and +1 can be expressed as

$$
\begin{aligned}
\operatorname{LLR}\left(x_{n}\right) & =\log \left(\frac{\operatorname{Pr}\left(r_{n} \mid x_{n}=1\right)}{\operatorname{Pr}\left(r_{n} \mid x_{n}=1\right)}\right) \\
& =\log \left(\frac{\exp \left(\frac{\left(1-r_{n}\right)^{2}}{-2 \sigma_{d}^{2}}\right)}{\exp \left(\frac{\left(-1-r_{n}\right)^{2}}{-2 \sigma_{d}^{2}}\right)}\right)=\frac{-4 r_{n}}{-2 \sigma_{d}^{2}} .
\end{aligned}
$$

The decision of errors may happen by hard decision when $r_{n}$ is close to 0 , i.e., $\operatorname{LLR}\left(x_{n}\right) \sim 0$. As a result, the unreliable region should be set to near zero value. Let $x_{n}=1$ be transmitted, then we have $r_{n} \sim N\left(1, \sigma_{d}^{2}\right)$, which is depicted in Fig. 3(a) and (b). For the detection scheme of a slice, when $r_{n}>0, x_{n}$ is detected as 1 ; otherwise, it is set to 0 . As we can see, when $r_{n}$ is close to 0 , the decision is very unreliable. Fig. 3(b) shows the detection scheme of the two-layer STM algorithm. $L$ is denoted as a threshold value between the reliable and unreliable regions. When $\left|r_{n}\right|<L, r_{n}$ is said to be in the unreliable region, we do not make the decision but forward the $\operatorname{LLR}\left(x_{n}\right)$ to the next symbol. We denote the operation of the STM-algorithm in Stage 1 at time $n$ and in Stage 2 at time $n+1$, as shown in Fig. 3. For the two-layer STM-algorithm, the last stage number is set to 2. Therefore, we must detect two symbols $\left\{x_{n+1}, x_{n}\right\}$ simultaneously in Stage2, and then, the stage changes into Stage 1. First, we explain the effect of $L$ as follows.

- $L$ is Larger: When $-L<r_{n}<0$, an error arises from a slicer. If we want to detect more errors, $L$ must be set at a larger value. As the threshold value $L$ is extended to be $L_{1}$ as shown in Fig. 3, both regions $S$ and $S$ ' are the incremental unreliable regions. At time $n$, a slicer has the

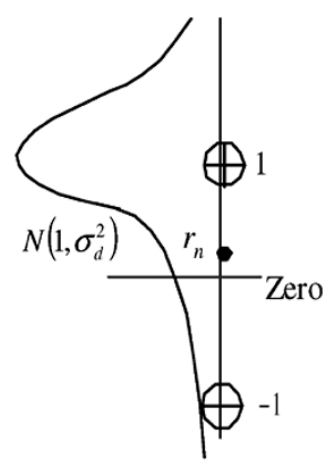

(a)

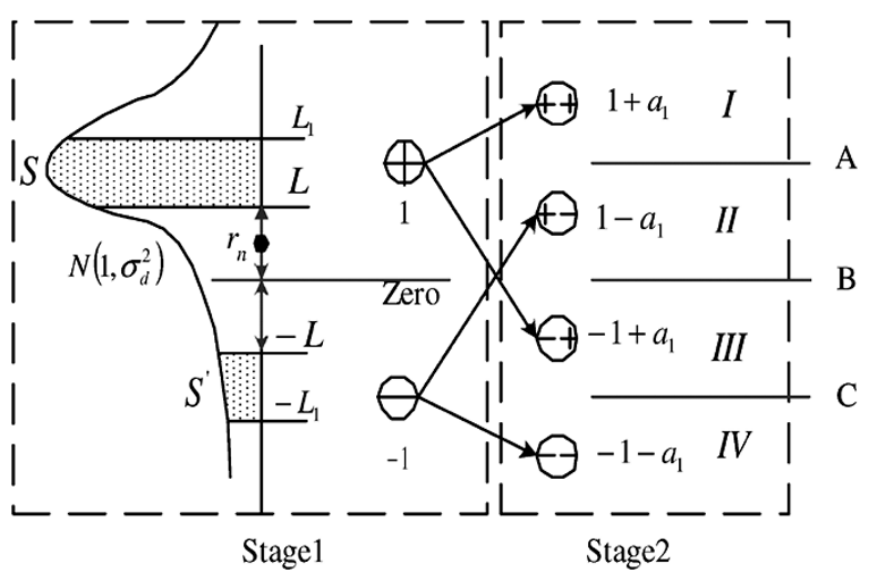

(b)

Fig. 3. Detection scheme. (a) Slice. (b) Two-layer STM-algorithm. The output $r_{n}$ of the STM algorithm may be 1 or -1 in Stage 1 . In Stage $2, r_{n+1}$ may be one of four values $\left(1+a_{1}, 1-a_{1},-1+a_{1}\right.$, or $\left.-1-a_{1}\right)$ since the transmitted data $x_{n}$ is undetermined at time $n$. Lines $A, B$, and $C$ are the boundaries of decision regions of these four values. $N(\bullet)$ is the normal distribution function

correct decision when $r_{n}$ is in region $S$; on the contrary, the decision by a slicer is wrong when $r_{n}$ is in region $S$ '. Since $r_{n} \sim N\left(1, \sigma_{d}^{2}\right)$, region $S^{\prime}$ is smaller than region $S$. Thus, if we enlarge $L$ to detect more errors, the more correct decisions by a slicer, i.e., region $S$, are sent to Stage 2 simultaneously and detected again. Unfortunately, the distance of states in Stage 2 is closer than that in Stage 1. That is, the detections tend to be erroneous when they are detected in Stage 2.

- $L$ is Smaller: For the smaller $L$, the less correct decisions by a slicer are set to Stage 2, but fewer errors will be detected.

Therefore, $L$ must be determined appropriately.

1) MAP Detection in Stage 2: The STM algorithm has the same decision scheme as the slicers in Stage 1 when $r_{\mathrm{n}}$ is reliable. Otherwise, the process of the STM algorithm changes into Stage 2. Next, we show how to detect the transmitted data and minimize the error probability in Stage 2. Then, we apply the results to figure out the optimal threshold value $L$. In Stage 2, we define

$$
\begin{aligned}
\bar{r}_{n+1} & =\sum_{m} b_{m} \times y_{n+1-m}-\sum_{m \neq 1} a_{m} \times \hat{x}_{n-m+1} \\
& =x_{n+1}+a_{1} x_{n}+\varepsilon_{n+1}^{d}
\end{aligned}
$$


where $\bar{r}_{n+1}$ is independent of $\hat{x}_{n}$ since the value of $x_{\mathrm{n}}$ is not determined at time $n$. In (5b), we apply the assumptions A2) and A3). We need to detect $x_{n}$ and $x_{n+1}$ after $r_{\mathrm{n}}$ and $\bar{r}_{n+1}$ are figured out. From (5b), when $\left\{x_{n+1}, x_{n}\right\}$ is $\{+1,+1\},\{+1,-1\},\{-1,+1\}$ and $\{-1,-1\}$, the value of states $\bar{r}_{n+1}$ are $1+a_{1}, 1-a_{1},-1+a_{1}$, and $-1-a_{1}$, respectively. We illustrate the states in Fig. 3(b). The conditional probability of $x_{n}$ and $x_{n+1}$ on $r_{n}$ and $\bar{r}_{n+1}$ at time $n+1$ can as in (6), shown at the bottom of the page.

Applying the property that $\bar{r}_{n+1}$ and $r_{n}$ are independent conditional on $x_{n}$ and $x_{n+1}$ in (6a), (6a) can be reformulated as (6b), which is proportional to

$$
\operatorname{Pr}\left(\bar{r}_{n+1} \mid x_{n+1}, x_{n}\right) \operatorname{Pr}\left(r_{n} \mid x_{n}\right) \text {. }
$$

Equation (7) is used to determine the boundaries of decision regions to minimize the probability of decision error in Stage 2.

2) Boundaries of Decision Regions in Stage 2: We will focus on $a_{1}>0$ because we can just change the sign of $x_{n}$ for Case $a_{1}<0$. The boundaries between regions $I-I V$ are labeled as $A, B$, and $C$ in Stage 2, as shown in Fig. 3. When $\bar{r}_{n+1}$ is in regions $I-I V,\left\{x_{n+1}, x_{n}\right\}$ is detected as $\{+1,+1\},\{+1,-1\},\{-1,+1\}$, and $\{-1,-1\}$, respectively. We derive the boundary value in the Appendix. To compute the probability of decision errors in Stage 2, three cases of boundary problems must be noted and described as below.

Case 1) $\left|r_{n}\right|<a_{1}\left(1-a_{1}\right)$ : In this normal case, three boundaries are $A=\left(1-r_{n} / a_{1}\right), B=\left(r_{n} /\left(1-a_{1}\right)\right)$, and $C=\left(-1-r_{n} / a_{1}\right)$.

Case 2) $r_{n}>a_{1}\left(1-a_{1}\right)$ : According to (7), the boundary value $A$ and $B$ are $\left(1-r_{n} / a_{1}\right)$ and $\left(r_{n} /\left(1-a_{1}\right)\right)$. However, when $r_{n}=a_{1}\left(1-a_{1}\right)$, both boundaries $A$ and $B$ are equal to $a_{1}$. In this case, region $I I$ vanished [see Fig. 4(a)]. When $\bar{r}_{n+1}$ is larger than $C=\left(-1-r_{n} / a_{1}\right), x_{\mathrm{n}}$ is detected as +1 . That is, $\left\{x_{n+1}, x_{n}\right\}$ cannot be detected as $\{+1,-1\}$. It results in $x_{\mathrm{n}}$ being detected as +1 with higher probability. Reasonably, $x_{\mathrm{n}}$ should have a larger probability of being detected as +1 in Stage 2, when $r_{\mathrm{n}}$ is closer to +1 .

Case 3) $r_{n}<-a_{1}\left(1-a_{1}\right)$ : This case is the opposite of Case 2), as illustrated by Fig. 4(b). When $r_{n}=$ $-a_{1}\left(1-a_{1}\right), \mathrm{A}=\left(1-r_{n} / a_{1}\right)$, and $\mathrm{B}=\mathrm{C}=-a_{1}$.

As shown in the above discussion, the STM-algorithm utilizes the information from $r_{n}$, which is not used in [6]:

- The information of $\operatorname{Pr}\left(r_{n} \mid x_{n}\right)$ is used at time $n+1$, which can make decisions more correct in Stage 2.

- The boundary of decision regions in Stage 2 is dynamically adjusted to be optimal based on $r_{n}$ and not a given constant value in [6].

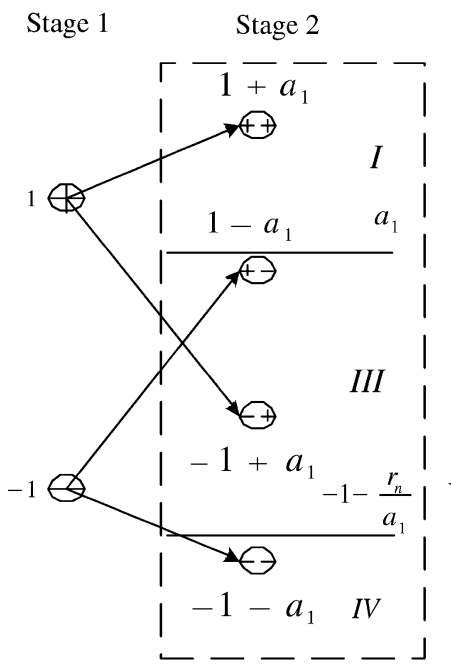

(a)

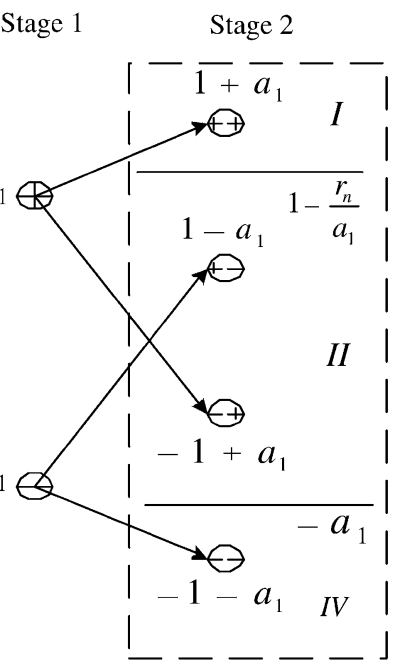

(b)
Fig. 4. Disappearance of the decision regions $I I$ and $I I I$ in Stage 2 of the STM-algorithm. Case a) for $\left|r_{n}\right| \geq a_{1}\left(1-a_{1}\right)$ and Case b) for $\left|r_{n}\right| \leq$ $a_{1}\left(1-a_{1}\right)$.

\section{B. Optimal Threshold Value L for Minimizing BER}

As in the previous discussion, the threshold value $L$ of the reliable/unreliable region is significant in the reduction of errors. In the two-layer STM algorithm, $L$ is chosen to maximize the number of the reduced decision errors by using the STM engine at times $n$ and $n+1$. In the discussion below, we assume that $r_{\mathrm{n}}$ is in the unreliable region at time $n$ and that $x_{\mathrm{n}}=+1$ is transmitted. By symmetry, $x_{\mathrm{n}}=-1$ can follow the same way.

1) Analysis of Decision Error by Using Slicers: At time $n$ and $n+1$, the expectation of decision errors by using a slicer is the summation of the two following cases.

Case 1) $0 \leq r_{\mathrm{n}} \leq L$

The slicer has the correct decision at time $n$. Therefore, only one error may happen at time $n+1$, and the expectation of decision errors is

$$
\begin{aligned}
\varepsilon_{\text {Slicer }, 1}(L)= & \int_{0}^{L} \int_{-\infty}^{0} q\left(r_{n}=s \mid x_{n}=1\right) \operatorname{Pr}\left(x_{n+1}=1\right) \\
& \times q\left(\bar{r}_{n+1}=t-1\right) d t d s \\
& +\int_{0}^{L} \int_{0}^{\infty} q\left(r_{n}=s \mid x_{n}=1\right) \operatorname{Pr}\left(x_{n+1}=-1\right) \\
& \times q\left(\bar{r}_{n+1}=t-1\right) d t d s
\end{aligned}
$$

where noise density function $q(l)$ is the normal distribution with zero mean and variance $\sigma_{d}^{2}$ by assumption A2), i.e., $q(l) \sim N\left(0, \sigma_{d}^{2}\right)$.

$$
\begin{gathered}
\operatorname{Pr}\left(x_{n+1}, x_{n} \mid \bar{r}_{n+1}, r_{n}\right)=\frac{\operatorname{Pr}\left(\bar{r}_{n+1}, r_{n} \mid x_{n+1}, x_{n}\right) \times \operatorname{Pr}\left(x_{n+1}, x_{n}\right)}{\operatorname{Pr}\left(\bar{r}_{n+1}\right) \times \operatorname{Pr}\left(r_{n}\right)} \\
\quad=\frac{\operatorname{Pr}\left(\bar{r}_{n+1} \mid x_{n+1}, x_{n}\right) \times \operatorname{Pr}\left(r_{n} \mid x_{n+1}, x_{n}\right) \times \operatorname{Pr}\left(x_{n+1}\right) \times \operatorname{Pr}\left(x_{n}\right)}{\operatorname{Pr}\left(\bar{r}_{n+1}\right) \times \operatorname{Pr}\left(r_{n}\right)} .
\end{gathered}
$$


Case 2) $-L \leq r_{\mathrm{n}} \leq 0$

In this case, the slicer makes the wrong decision at time $n$. Then, the error will propagate and cause an offset in next symbol. Applying Assumption A3), the sum of the expectation of decision errors at time $n$ and $n+1$ is

$$
\begin{aligned}
& \varepsilon_{\text {Slicer }, 2}(L) \\
& =\int_{-L}^{0} q\left(r_{n}=s \mid x_{n}=1\right) \\
& \quad \times\left\{\operatorname { P r } ( x _ { n + 1 } = 1 ) \left[2 \int_{-\infty}^{2 a_{1}} q\left(\bar{r}_{n+1}=t-1\right) d t+1\right.\right. \\
& \left.\quad \times\left(1-\int_{-\infty}^{2 a_{1}} q\left(\bar{r}_{n+1}=t-1\right) d t\right)\right] \\
& \quad+\operatorname{Pr}\left(x_{n+1}=-1\right)\left[2 \int_{-2 a_{1}}^{\infty} q\left(\bar{r}_{n+1}=t-1\right) d t+1\right. \\
& \left.\left.\quad \times\left(1-\int_{-2 a_{1}}^{\infty} q\left(\bar{r}_{n+1}=t-1\right) d t\right)\right]\right\} d s .
\end{aligned}
$$

Therefore, the expectation of total decision errors made by a slicer at time $n$ and $n+1$ is the summation of (8)and (9) and is denoted as

$$
\varepsilon_{\text {Slicer }}(L)=\varepsilon_{\text {Slicer }, 1}(L)+\varepsilon_{\text {Slicer }, 2}(L) .
$$

2) Analysis of Decision Error by Using the STM-Engine: Next, we will figure out the expectation of decision errors by the STM engine. In addition, we assume $x_{n}=+1$, and $r_{n}$ is in the unreliable region.

Case 1) When $x_{n+1}$ is +1 , the STM-engine makes one or two bit errors if it determines $\left\{x_{n+1}, x_{n}\right\}$ to be $\{+1,-1\}$ and $\{-1,+1\}$, or $\{-1,-1\}$, respectively. The expectation of decision errors can be formulated as

$$
\begin{aligned}
& \varepsilon_{\mathrm{STM}, 1}(L) \\
& =\int_{-L}^{L} \operatorname{Pr}\left(r_{n}=s \mid x_{n}=1\right)\left\{\operatorname{Pr}\left(x_{n+1}=1\right)\right. \\
& \quad \times\left[\int_{\text {region II }+\mathrm{III}} q\left(\bar{r}_{n+1}=t-1-a_{1}\right) d t+2\right. \\
& \left.\left.\quad \times \int_{\text {region IV }} q\left(\bar{r}_{n+1}=t-1-a_{1}\right) d t\right]\right\} d s .
\end{aligned}
$$

Case 2) When -1 is transmitted at time $n+1$, the STM-algorithm makes one or two bit errors if $\left\{x_{n+1}, x_{n}\right\}$ is chosen as $\{+1,+1\}$ (State $I$ ) and $\{-1,-1\}$ (State
$I V)$, or $\{-1,+1\}$ (State III), respectively. The expectation of decision errors is

$$
\begin{aligned}
& \varepsilon_{\mathrm{STM}, 2}(L) \\
& =\int_{-L}^{L} \operatorname{Pr}\left(r_{n}=s \mid x_{n}=1\right)\left\{\operatorname{Pr}\left(x_{n+1}=-1\right)\right. \\
& \times\left[\int_{\text {region I }+\mathrm{IV}} q\left(\bar{r}_{n+1}=t+1-a_{1}\right) d t+2\right. \\
& \left.\left.\quad \times \int_{\text {region III }} q\left(\bar{r}_{n+1}=t+1-a_{1}\right) d t\right]\right\} d s .
\end{aligned}
$$

The expectation of total decision errors by the STMalgorithm is

$$
\varepsilon_{\mathrm{STM}}(L)=\varepsilon_{\mathrm{STM}, 1}(L)+\varepsilon_{\mathrm{STM}, 2}(L) .
$$

3) Solution of the Optimal Threshold Value: From (8) and (13), the STM-engine reduces the decision errors by $\varepsilon_{\text {Slicer }}(L)-$ $\varepsilon_{\mathrm{SML}}(L)$, which is a function of $L$. When $L$ satisfies

$$
\left.\frac{\partial}{\partial L}\left(\varepsilon_{\text {Slicer }}(L)\right)-\varepsilon_{\mathrm{STM}}(L)\right)=0
$$

the STM engine achieves the maximum reduction of errors. For $L \leq a_{1}\left(1-a_{1}\right)$, the optimal threshold value $L$ must satisfy

$$
\begin{aligned}
& \exp \left(\frac{(1-L)^{2}}{2 \sigma_{d}^{2}}\right)\left[2 \times Q\left(\frac{a_{1}+L / a_{1}}{\sigma_{d}}\right)+2\right. \\
& \quad \times Q\left(\frac{1-a_{1}+L /\left(1-a_{1}\right)}{\sigma_{d}}\right)+Q\left(\frac{2+a_{1}+L / a_{1}}{\sigma_{d}}\right) \\
& \left.-Q\left(\frac{2-a_{1}-L / a_{1}}{\sigma_{d}}\right)-2 \times Q\left(\frac{1}{\sigma_{d}}\right)\right] \\
& \quad+\exp \left(\frac{(1+L)^{2}}{2 \sigma_{d}^{2}}\right)\left[2 \times Q\left(\frac{a_{1}-L / a_{1}}{\sigma_{d}}\right)+2\right. \\
& \quad \times Q\left(\frac{1-a_{1}-L /\left(1-a_{1}\right)}{\sigma_{d}}\right)+Q\left(\frac{2+a_{1}-L / a_{1}}{\sigma_{d}}\right) \\
& -Q\left(\frac{2-a_{1}+L / a_{1}}{\sigma_{d}}\right)-Q\left(\frac{1+2 a_{1}}{\sigma_{d}}\right) \\
& \left.-Q\left(\frac{1-2 a_{1}}{\sigma_{d}}\right)-2\right]=0 .
\end{aligned}
$$

For $L \geq a_{1}\left(1-a_{1}\right), L$ must satisfy

$$
\begin{gathered}
\exp \left(\frac{(1-L)^{2}}{2 \sigma_{d}^{2}}\right)\left[Q\left(\frac{a_{1}+L / a_{1}}{\sigma_{d}}\right)+Q\left(\frac{2+a_{1}+L / a_{1}}{\sigma_{d}}\right)\right. \\
\left.-Q\left(\frac{2-a_{1}-L / a_{1}}{\sigma_{d}}\right)-Q\left(\frac{1}{\sigma_{d}}\right)\right]+\exp \left(\frac{(1+L)^{2}}{2 \sigma_{d}^{2}}\right) \\
\quad \times\left[Q\left(\frac{a_{1}-L / a_{1}}{\sigma_{d}}\right)-Q\left(\frac{2-a_{1}+L / a_{1}}{\sigma_{d}}\right)-1\right]=0
\end{gathered}
$$

where $Q(x) \equiv \int_{x}^{\infty} e^{-x^{2} / 2} d x$. The threshold $L$ of the reliable/unreliable region is a function of $a_{1}$ and $\sigma_{d}$. For VLSI implementations, $L$ must be updating adaptively along with $a_{1}$ and 
$\sigma_{d}$. Fortunately, $L$ can be approximated as $a_{1}\left(1-a_{1}\right)$. We show this with numerical results in Sections IV-A and C.

\section{Higher Order Layer of the STM Algorithm}

In the TT-DFE, the error correction scheme is based on two successive symbols. It is difficult to extend this scheme to more than two successive symbols. However, the proposed STM-Algorithm is easy to extend to higher layers. For the derivation of the $N$-layer STM algorithm, we follow the derivation of the two-layer STM algorithm. In Stage $N$, we define

$$
\begin{aligned}
\bar{r}_{n+N-1}= & \sum_{m} b_{m} \times y_{(n+N-1)-m} \\
& -\sum_{m \neq 1,2, \ldots, N-1} \hat{x}_{(n+N-1)-m} \\
= & x_{n+N-1}+a_{1} x_{n+N-2}+a_{2} x_{n+N-3}+\cdots \\
& +a_{N-1} x_{n}+\varepsilon_{n+N-1}^{d} .
\end{aligned}
$$

The detection at Stage $N$ can be formulated as

$$
\begin{aligned}
\operatorname{Pr}( & \left.x_{n+N-1}, x_{n+N-2}, \ldots, x_{n} \mid \bar{r}_{n+N-1}, \bar{r}_{n+N-2}, \ldots, r_{n}\right) \\
= & \operatorname{Pr}\left(\bar{r}_{n+N-1} \mid x_{n+N-1}, x_{n+N-2}, \ldots, x_{n}\right) \\
& \times \operatorname{Pr}\left(\bar{r}_{n+N-2} \mid x_{n+N-2}, x_{n+N-1}, \ldots, x_{n}\right) \times \cdots \\
& \times \operatorname{Pr}\left(r_{n} \mid x_{n}\right) \\
& \times \frac{\operatorname{Pr}\left(x_{n+N-1}\right) \times \operatorname{Pr}\left(x_{n+N-1}\right) \times \cdots \times \operatorname{Pr}\left(x_{n}\right)}{\operatorname{Pr}\left(\bar{r}_{n+N-1}\right) \times \operatorname{Pr}\left(\bar{r}_{n+N-1}\right) \times \cdots \times \operatorname{Pr}\left(r_{n}\right)} .
\end{aligned}
$$

Equation (18) can be recursively computed, and only $\operatorname{Pr}\left(\bar{r}_{n+N-1} \mid x_{n+N-1}, x_{n+N-2}, \ldots, x_{n}\right)$ needs to be calculated in Stage $N$. Other parts can be referred to log-likelihood. They are calculated in Stages $1,2, \ldots$, and $N-1$. Moreover, in implementing an equalizer, the performance of the BER as well as the hardware cost must be taken into account. To attain higher performance of the BER, one way is to extend the STM-DFE to a higher order layer. We depict the simple concept in Fig. 5(a). However, the number of states is exponential to the number of stages. Extending to the $N$-layer STM-DFE, the state number of the trellis diagram will expand up to $2^{n}$. This places high demand on the hardware. We can limit our search range in higher probability regions to reduce the state number. Take the three-layer STM for example; when $\bar{r}_{n+1}$ is around line $A$ [see Fig. 5(b)], then the probability of state 1 and 2 is higher than states 3 and 4 . In this case, we set $\left\{x_{n+1}, x_{n}\right\}$ to be $\{+1,+1\}$ or $\{+1,-1\}$. Furthermore, in Stage 3, we just begin from states 1 and 2 in Stage 2 (solid line in Fig. 5(b)). The other two cases can follow in the same way. Therefore, the number of states always remains in 4, where they do not grow exponentially with the number of stages.

In the STM algorithm, we can find the optimal threshold value off-line and approximate it with a polynomial function so that the threshold value can be solved in running time. To solve the threshold, it is very complicated to figure out the threshold values in all stages simultaneously. Our scheme is to find the threshold values $1,2, \ldots, k-1$ and then use the $k-1$ value to figure out the next $k$. We take the three-layer as an example. In

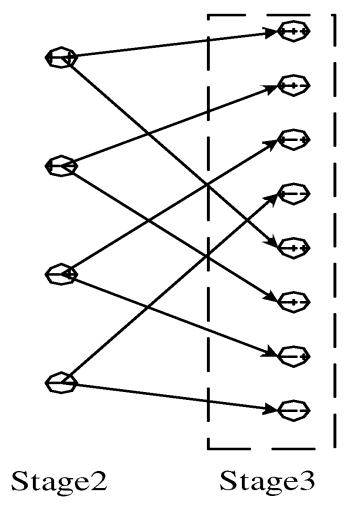

(a)

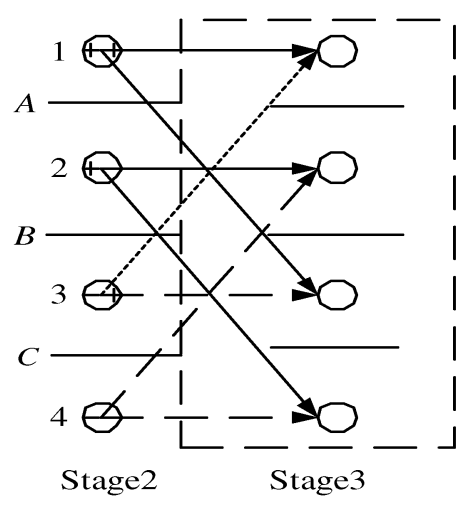

(b)
Fig. 5. (a) Extended trellis diagram of the three-layer STM-algorithm in which we do not apply the limited searching scheme. (b) Reduced trellis diagram when we apply the limited searching scheme.

Section III-B, we have solved the threshold value in Stage 1. To solve the threshold values in Stage 2, first, we use (18) to find the boundaries of the decision regions in Stage 3. Next, using the method in Section III-B, we apply these boundaries and the threshold value $L$ in Stage 1 to figure out the threshold values of Stage 2. In Stage 2, there are three thresholds around lines $A, B$, and $C$, respectively. Due to the symmetry, the thresholds near lines $A$ and $C$ are equal. To list these equations is lengthy and tedious. We do not list the equations but show them by simulations. To compute the threshold values of the $N$-layer STM-algorithm, the steps are described as follows.

Step 1) Initially, $m$ is set to 1.

Step 2) (Boundary of Decision Regions): Based on (18), we first figure out the boundaries of decision regions in Stage $m+1$.

Step 3) (Threshold Value Determination): We follow the derivation in Section III-B and apply the threshold values in Stage $1, \ldots$, and $m-1$ to find the threshold values in Stage $m$.

Step 4) Go to Step 2 when $m<N$.

\section{Stage Transition of the STM Algorithm}

Initially, the operation of the STM algorithm is in Stage 1, until the output of equalizers becomes unreliable, at which point, the stage changes into Stage 2; otherwise, the stage remains in Stage 1. This process can be continued. That is, if the received signal is unreliable in Stage $k$, the stage changes into Stage $k+1$; otherwise, the stage goes into Stage 1. I addition, we can limit the last stage number to a constraint constant $N_{s}$. We define the $N$-layer STM algorithm as its maximum stage number being $N$. Note that when one symbol is in the end of the feedback tap delay line, it will have no affect on the next output of the equalizers; therefore, the maximum stage number must be equal to or smaller than the tap number of FBF.

Fig. 6 shows the Stage transformation of the four-layer STM algorithm. When the output of equalizers is in the unreliable region in Stage $k<4$, we do not make the decision but forward the log-likelihood ratio to Stage $k+1$. We continue this process until the decision is reliable or the stage $k$ is 4 . Then, we must detect the transmitted data, and the stage changes into Stage 1. 


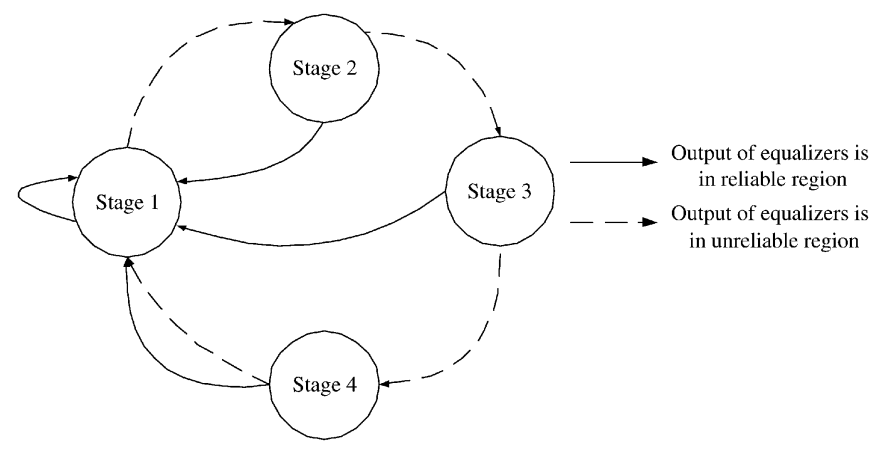

Fig. 6. Stage transition of the four-layer STM-algorithm.

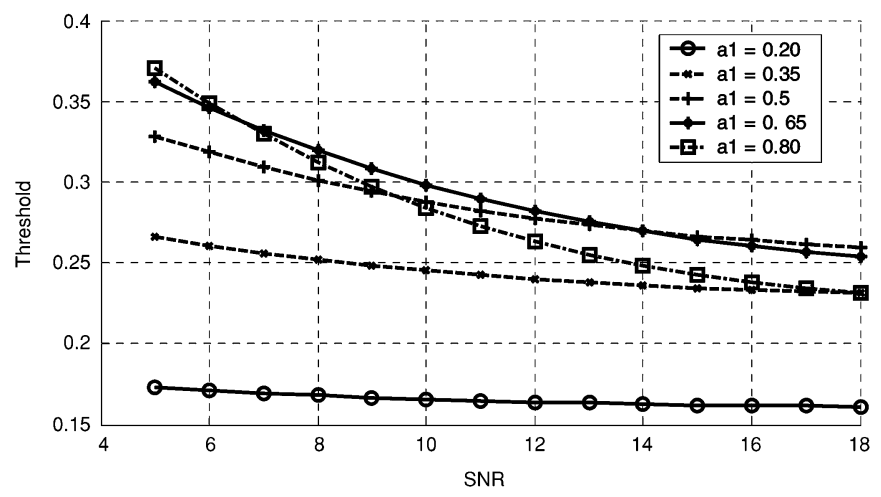

Fig. 7. Character of $L$ for different first tap weight of DFE.

\section{Numerical ANALYSIS AND SimUlation ResUlts}

In this section, we show the threshold value related to the first tap weight of the STM-DFE by the numerical result. To appreciate the effect of the STM-DFE, we compare it with the conventional DFE, TT-DFE, and IDFE for BER and BEL by simulations.

\section{A. Determination of Threshold Value}

Fig. 7 shows that all thresholds are larger under low SNR. This is very clear when the noise is larger; the log-likelihood ratio of $\operatorname{Pr}\left(x_{\mathrm{n}}=+1\right) / \operatorname{Pr}\left(x_{\mathrm{n}}=-1\right)$ is close to 1 in the larger region. That is, there is a larger unreliable region in low SNR. When $a_{1}<0.5$, the threshold value is close to $a_{1}\left(1-a_{1}\right)$ within a large range of high SNR. For case $a_{1}>0.5$, the threshold value is close to one above $a_{1}\left(1-a_{1}\right)$. Furthermore, when the output of the equalizers is in the unreliable range between state $2(+1,-1)$ and state $3(-1,+1)$, we may make 2-bit errors if the decision to be in state 2 or 3 is wrong. However, we make a 1-bit error in other cases. On the other hand, the distance of states 2 and 3 will become longer as $a_{1}$ becomes larger. This is the reason why when $a_{1}>0.5$, the threshold value is larger.

\section{B. Comparison of BER}

The channel model used is a Lorentzian channel at user density $\beta=2.5$ [7], which is common used in magnetic recording. All simulations have an average of 20 runs, and 100 million bits are used in each run. Designed equalizers are targeted at $\mathrm{SNR}=24 \mathrm{db}$. We assume the data fed back into the FBF are

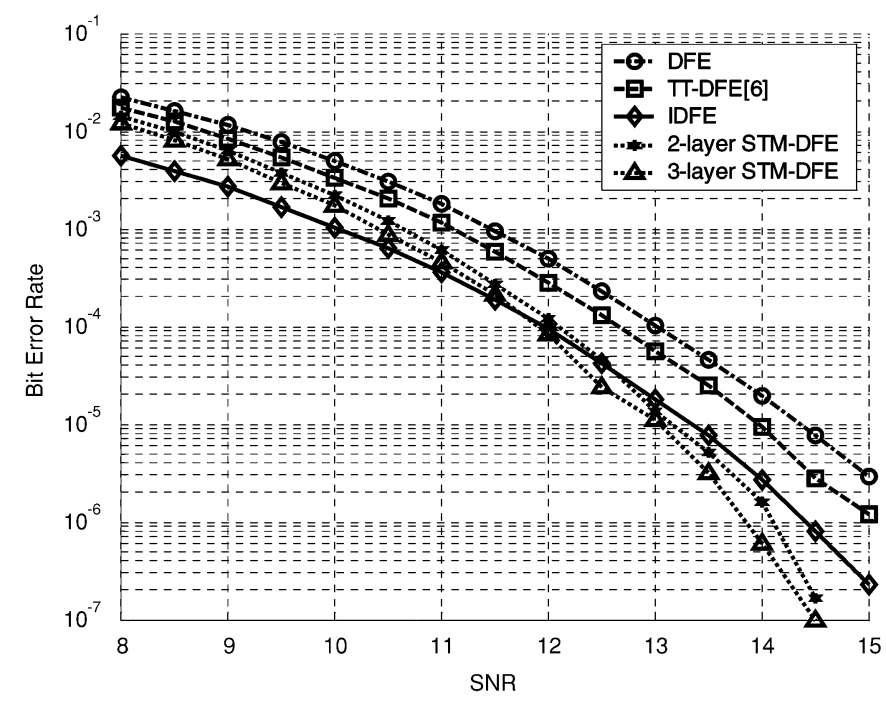

Fig. 8. Threshold of Stage 1 is approximated by $a 1(1-a 1)$. The parameters of TT-DFE are the same as [6].

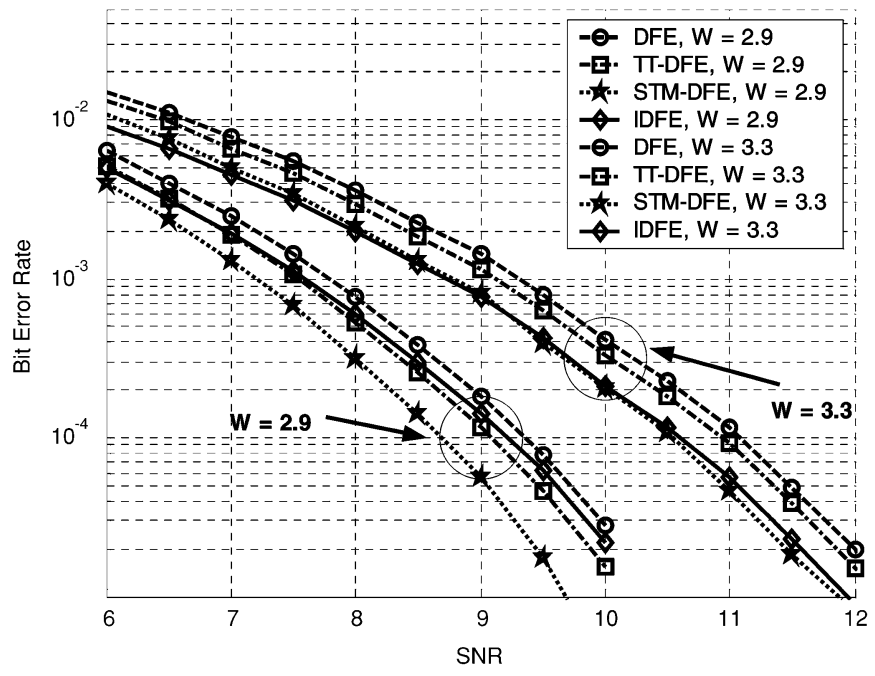

Fig. 9. Comparison of symbol error rate among DFEs and the STM-DFE. All cases average 20 runs. Each run has $5 \times 10^{8}$ symbols.

always correct in the case of the IDFE in order to measure the SNR degradation of DFE due to the error propagation. As shown in Fig. 8, the proposed STM-DFE has better performance than the DFE and TT-DFE. Moreover, when the SNR is larger than $12.5 \mathrm{db}$, the STM-DFE performs better than the IDFE. This is true even if the IDFE does not suffer from error propagation; it has no ability to detect and correct errors, which the STM-DFE can do, especially at higher SNR.

In the next scenario, the channel model is $\mathrm{h}=0.5[1+$ $\cos (2 \pi / \mathrm{w}) 21+\cos (2 \pi / \mathrm{w})][8]$. We use this to show the effects of the eigenvalue spectrum of the signal on the STM-algorithm. The number of taps in FFF and FBF are 7 and 5, respectively. Again, the number of errors corrected by the STM algorithm is larger at high SNR. The proposed algorithm can achieve the best SNR performance in two cases, as shown in Fig. 9. For the case of the smaller eigenvalue spectrum, the STM algorithm can outperform the IDFE in both low and high SNRs. For the case of $\mathrm{W}=3.3$, the STM-algorithm can achieve better results 


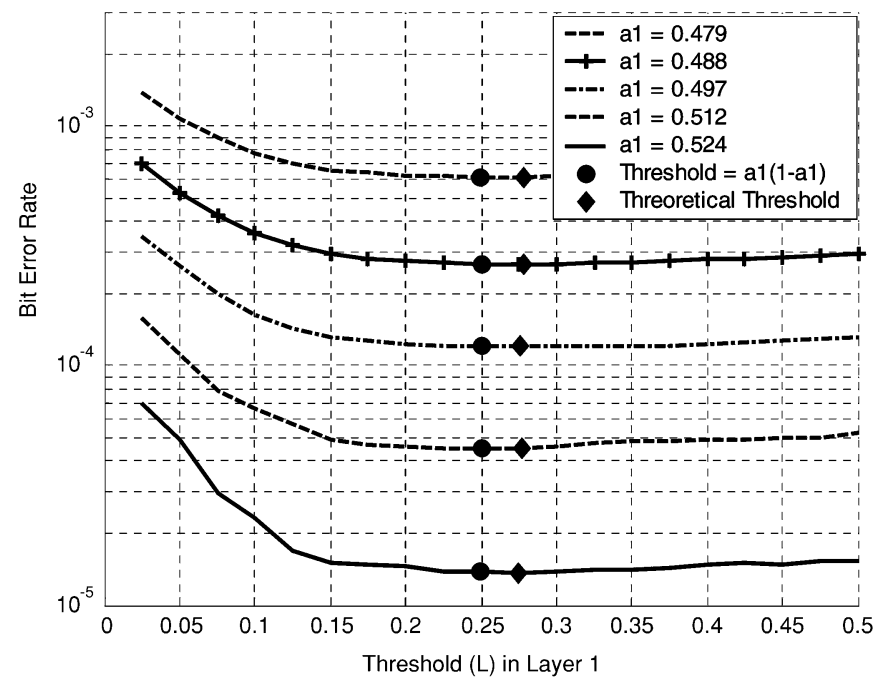

Fig. 10. Sensitivity due to the approximation of threshold by $L=a_{1}\left(1-a_{1}\right)$.

as the SNR becomes larger, and it has better performance than the IDFE from SNRs above $10 \mathrm{db}$.

\section{SNR Degradation Due to the Approximation of Threshold Value}

The configurations of the scenario are the same as that for the Lorentzian channel. As illustrated in Fig. 10, the theoretical analysis of the optimal threshold is consistent with simulation results in all cases. Furthermore, in order to figure out the threshold value, we must solve (15) and (16). However, in VLSI implementation, it is very complicated to solve these equations. The threshold is the function of $a_{1}$ and $\boldsymbol{\sigma}_{d}$. In general, we can approximate the threshold value via a polynomial function under the least square criterion. Then, the multipliers and adders are enough to produce the results. As the order of polynomial becomes higher, we can have better approximations. However, it will cost more in terms of hardware. As previously mentioned in Section III-A, we can approximate the threshold value by $a_{1}\left(1-a_{1}\right)$, which is the limited value in the boundary problems, as discussed in Section III-A. Therefore, the hardware cost to calculate the threshold value is just one adder and one square. As shown in Fig. 10, the curve is flat near the optimal value, and $a_{1}\left(1-a_{1}\right)$ is close to the optimum. Therefore, the approximation is very good in each case.

\section{Comparison of BEL}

The simulation setup is the same as Fig. 8. IDFE almost has no burst errors in our simulations. Fig. 11 shows that the proposed STM-DFE always achieves lower error probability than DFE and TT-DFE in every burst error length. In other words, the proposed algorithm performs better in reducing the error propagation. Moreover, the STM-DFE has the least probability of single error, even when the BER of the STM-DFE is larger than that of the IDFE. This is because the STM algorithm can efficiently correct the error, which is in the unreliable region in Stage 1. In addition, the three-layer STM-DFE has better performance than the two-layer STM-DFE for both BER and BEL.

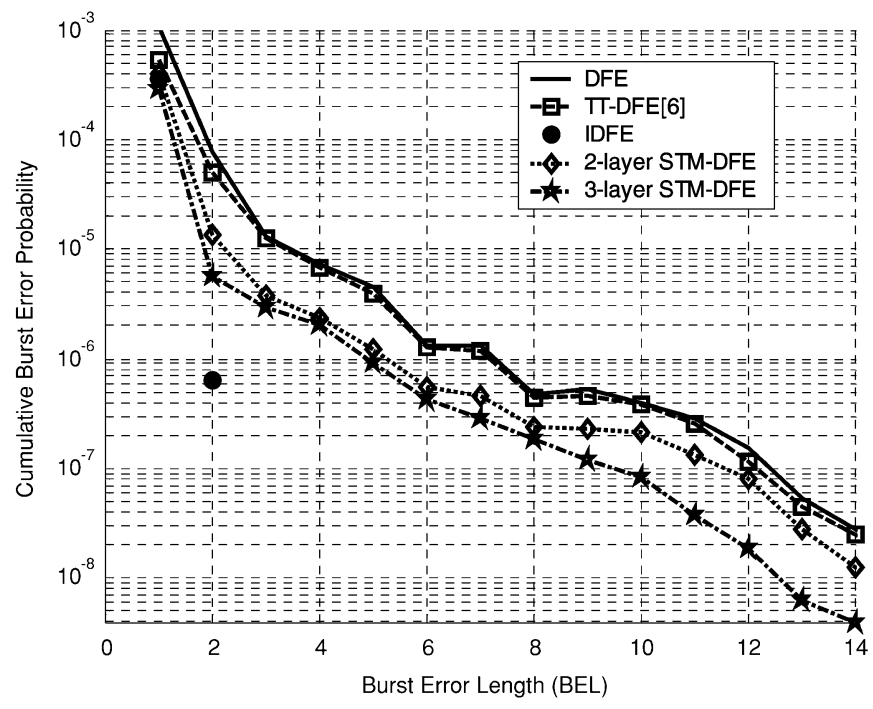

Fig. 11. BEL Comparison of SNR $=12.5 \mathrm{~dB}$.

\section{STM-DFE VLSI ARCHITECTURE}

\section{A. Algorithm Simplified for Low-Cost Implementation}

The simplified two-layer STM-DFE architecture is discussed in this section. We show the prototype of the two-layer STM engine in Fig. 12(a), which is the modified decision device. Two operation modes of the STM engine are described as follows:

1) Slicing Mode (SM): In this mode, the STM engine is in Stage 1 . The output of the equalizers is tested. If it is determined to be in the unreliable region by the threshold device, the STM engine works as a slicer and outputs the decision. Otherwise, the decision is delayed and the STM engine outputs a log-likelihood ratio. The operation mode changes into the delay decision mode (DDM).

2) Delay Decision Mode (DDM): In this mode, the STM engine detects two transmitted data and changes into the SM.

Next, we implement the STM algorithm in a simple way. As mentioned above, a slicer is the only required module in the SM. For the clarification of presentation, we assume that the transmitted data is 2-PAM. Based on the STM algorithm, $\left\{x_{n+1}, x_{n}\right\}$ are chosen to maximize (7). Applying the strict convex property of $\log$ function, $\left\{x_{n+1}, x_{n}\right\}$ can be chosen to maximize

$$
\begin{aligned}
& \log \left(\operatorname{Pr}\left(\bar{r}_{n+1} \mid x_{n+1}, x_{n}\right) \times \operatorname{Pr}\left(r_{n} \mid x_{n}\right)\right) \\
& \quad=\log \left(\operatorname{Pr}\left(\bar{r}_{n+1} \mid x_{n+1}, x_{n}\right)\right)+\log \left(\operatorname{Pr}\left(r_{n} \mid x_{n}\right)\right) \\
& \quad=\frac{\left(x_{n+1}-a_{1} x_{n}-\bar{r}_{n+1}\right)^{2}+\left(x_{n}-r_{n}\right)^{2}}{-2 \sigma_{d}^{2}}
\end{aligned}
$$

As a result, to find the maximum value of (19) is to minimize

$$
\left(x_{n+1}-a_{1} x_{n}-\bar{r}_{n+1}\right)^{2}+\left(x_{n}-r_{n}\right)^{2} .
$$

Therefore, the variance of error $\varepsilon_{d}$ is not involved in determining $\left\{x_{n+1}, x_{n}\right\}$. To figure out the solution, $\left\{x_{n+1}, x_{n}\right\}=$ $\{ \pm 1,+1\}$ and $\{ \pm 1,-1\}$ are taken into (20). Next, the smaller of each are compared to obtain the smallest. Based on (20), we require six square operations, 14 adders, and three comparators 


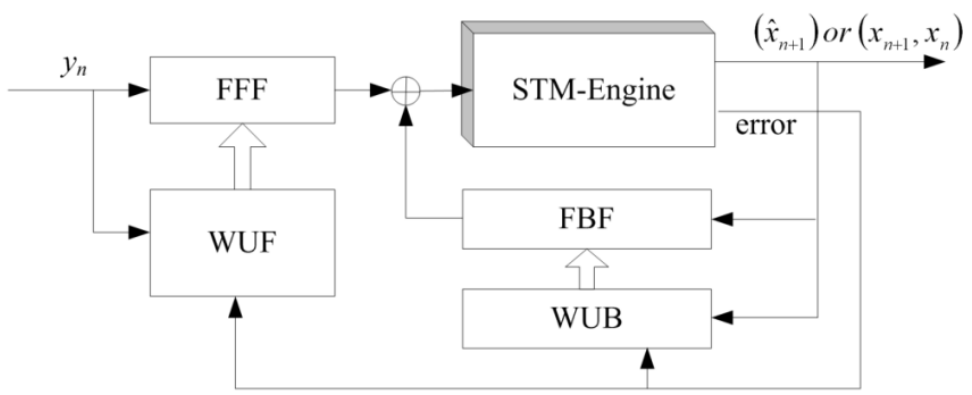

(a)

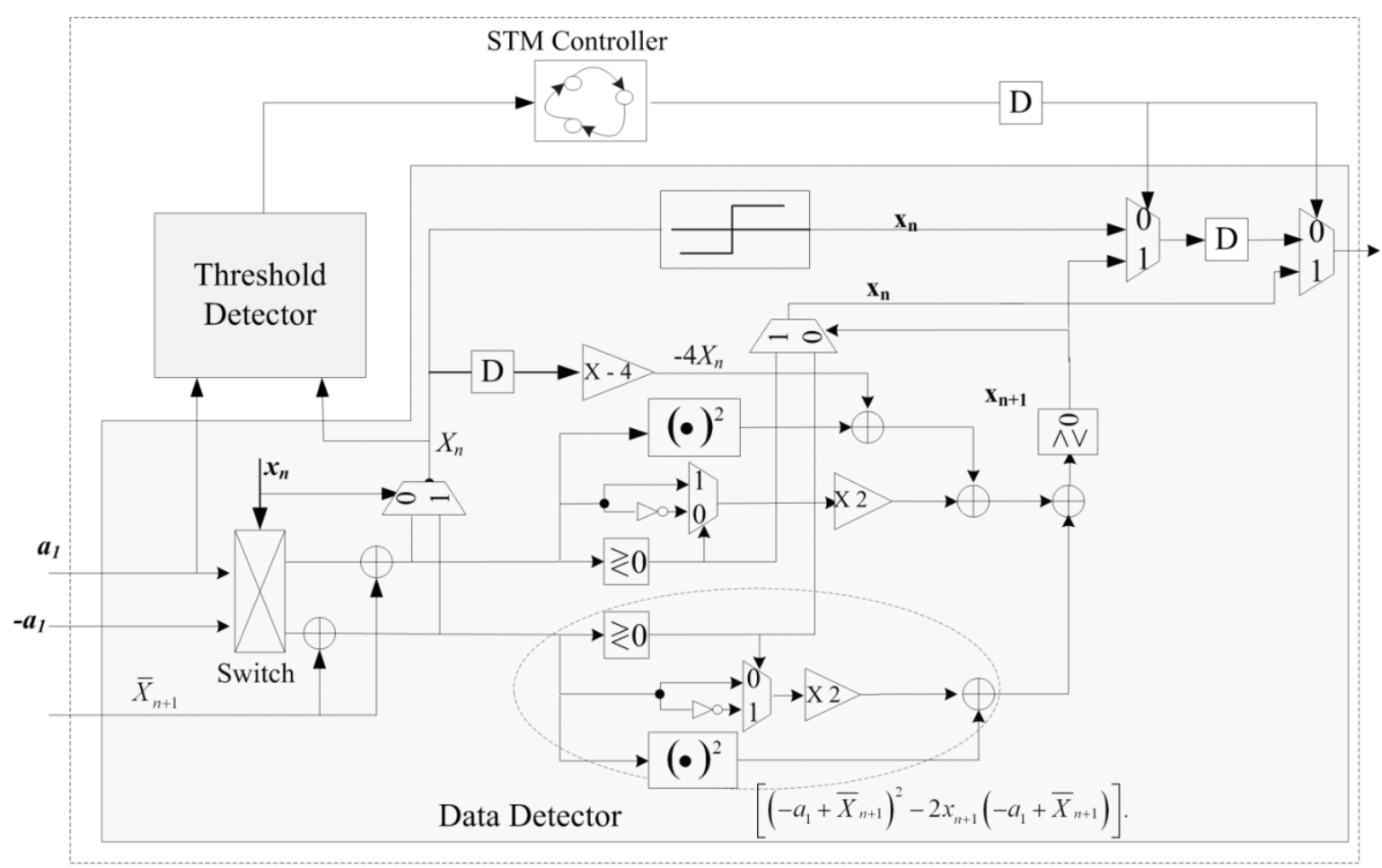

STM - Engine

(b)

Fig. 12. VLSI architecture of the two-layer STM-DFE engine. (a) Overall STM-DFE architecture. The block WUF and WUB is weight updating blocks for FFF and FBF, respectively. (b) STM engine.

to figure out the solution. To reduce the hardware complexity, the solution can be computed as follows:

Case 1) For $x_{\mathrm{n}}=1$

$$
\begin{gathered}
\left(x_{n+1}-a_{1} x_{n}-\bar{r}_{n+1}\right)^{2}+\left(x_{n}-r_{n}\right)^{2}=\left(2+r_{n}^{2}+2 r_{n}\right)^{2} \\
+\left[\left(a_{1}+\bar{r}_{n+1}\right)^{2}-2 x_{n+1}\left(a_{1}+\bar{r}_{n+1}\right)\right]^{2}-4 r_{n} .
\end{gathered}
$$

Case 2) For $x_{\mathrm{n}}=-1$

$$
\begin{aligned}
& \log \left(\operatorname{Pr}\left(\bar{r}_{n+1} \mid x_{n+1}, x_{n}=-1\right)\right)=\left(2+r_{n}^{2}+2 r_{n}\right)^{2} \\
& +\left[\left(-a_{1}+\bar{r}_{n+1}\right)^{2}-2 x_{n+1}\left(-a_{1}+\bar{r}_{n+1}\right)\right] .
\end{aligned}
$$

Moreover, the term $\left(2+r_{n}^{2}+2 r_{n}\right)$ can be removed from (21) and (22), and $\left(-4 r_{n}\right)$ is added into (21), which is given by Stage 1. Consequently, when $\left(a_{1}+\right.$ $\left.\bar{r}_{n+1}\right)>0$ for case $x_{n}=1$ or $\left(-a_{1}+\bar{r}_{n+1}\right)>0$ for case $x_{n}=-1, x_{n+1}$ should be selected as 1. Otherwise, $x_{n+1}$ is set to -1 . To determine $x_{n}$, the two smaller ones of each of the cases above are compared with each other.

\section{B. VLSI Architecture of the STM-DFE}

As shown in the discussion in Section V-A, we need only two square operations and five adders. The STM-DFE controller can be implemented by a simple two-state finite state machine (FSM). We show the prototype of the two-layer STM engine architecture in Fig. 12. In [14] and [15], the square operations are designed to be smaller in area and higher in computing speed than one multiplier. In summary, the major hardware overhead is only three square operations, as well as six additions and one switch. 
TABLE I

HaRdware Complexity COMPARISON. $N_{a}$ AND $N_{b}$ ARE THE TAP Size of FBF AND FFF, ResPeCtively. THE LMS ALGORITHM Is USED TO UPDATE THE WEIGHT COEFFICIENTS

\begin{tabular}{c|c|c|c|c|c}
\hline Algorithm & $\begin{array}{c}\text { Number of } \\
\text { Multipliers }\end{array}$ & $\begin{array}{c}\text { Number of } \\
\text { Adders }\end{array}$ & $\begin{array}{c}\text { Threshold } \\
\text { Detector }\end{array}$ & $\begin{array}{c}\text { Number of } \\
\text { Squares }\end{array}$ & $\begin{array}{c}\text { Number of Switches } \\
\text { and Multiplexers }\end{array}$ \\
\hline $\begin{array}{c}\text { Conventional } \\
\text { DFE }\end{array}$ & $2\left(N_{a}+N_{b}\right)$ & $2\left(N_{a}+N_{b}\right)$ & 0 & 0 & 0 \\
\hline TT-DFE & $2\left(N_{a}+N_{b}\right)$ & $2\left(N_{a}+N_{b}\right)$ & 2 & 0 & 0 \\
\hline STM-DFE & $2\left(N_{a}+N_{b}\right)$ & $2\left(N_{a}+N_{b}\right)+6$ & 1 & 3 & 1 Switch \\
\hline $\begin{array}{c}\text { 1-stage } \\
\text { look-ahead } \\
\text { STM-DFE }\end{array}$ & $2\left(N_{a}+N_{b}\right)$ & $2\left(N_{a}+N_{b}\right)+8$ & 1 & 3 & 2 Multiplexers \\
+1 Switch
\end{tabular}

Moreover, the STM-DFE will increase the latency in DFL. $T_{S}, T_{A}, T_{\mathrm{FA}}$, and $T_{\mathrm{Sw}}$ denote the latency of square, adders, one-bit full adder, and switch operations, respectively. As shown in Fig. 12, the increasing latency of the two-layer STM-DFE is $\left(T_{S}+3 \times T_{A}+T_{\mathrm{Sw}}\right)$. Generally, the area/latency of adders and square operators are dependent on hardware architectures. In this paper, we implement square operators and adders with array multipliers and ripple adders, respectively. For a wordlength $W, T_{S}$ and $T_{A}$ are $(2 W-1) \times T_{\mathrm{FA}}$ and $W \times T_{\mathrm{FA}}$, respectively. Therefore, $T_{S}$ is about twice $T_{A}$. To estimate the overhead due to increased latency of the STM engine, we apply the STM algorithm to the Lorentzian channel. The tap number $N_{a}$ is set as 7 to achieve BER $=10^{-5}$ at SNR $=14 \mathrm{db}$. As a result, the latencies of the STM engine and conventional DFE are $\left(T_{S}+10 \times T_{A}+T_{\mathrm{Sw}}\right)$ and $\left(7 \times T_{A}\right)$, respectively. Thus, the latency of the STM engine is about twice that of the conventional DFE. As a result, the one-stage pipelined STM can operate at the same clock rate as the conventional DFE (the one-stage pipelined STM will be discussed in the next section). This will require two adders and two 2-to-1 multiplexer overheads, as shown in Fig. 14. Table I summarizes the hardware complexity.

\section{Pipelined STM-DFE (PSTM-DFE) Architecture}

In practical equalizer designs of high-speed application systems, the computing speed is one of the main issues. Delay LMS (DLMS) [10] is a very low-cost scheme to reduce the critical path and speed up the rate of computing. However, the performance, such as SNR or convergence rate, will be degraded severely by employing this method. In our approach, we employ the look-ahead scheme of [10] and [13] to pipeline our STM-DFE equalizer. Fig. 13 shows the two-stage look-ahead. We have two extra delays to pipeline the feedback loop. To pipeline the STM-DFE, two modifications are made as follows:

1) Move the slicer to the front of the multiplexers: If we substitute slicers with STM engines, the overhead will increase dramatically. Hence, we first move the slicer to the front of the multiplexers. Next, the slicer is substituted by the STM engine, as in Fig. 14. The overhead of the STM-DFE is the same as that of the conventional DFE.

2) Look-ahead of tap-weight: The signal fed back into the first tap of FBF is unknown in the delay decision mode;

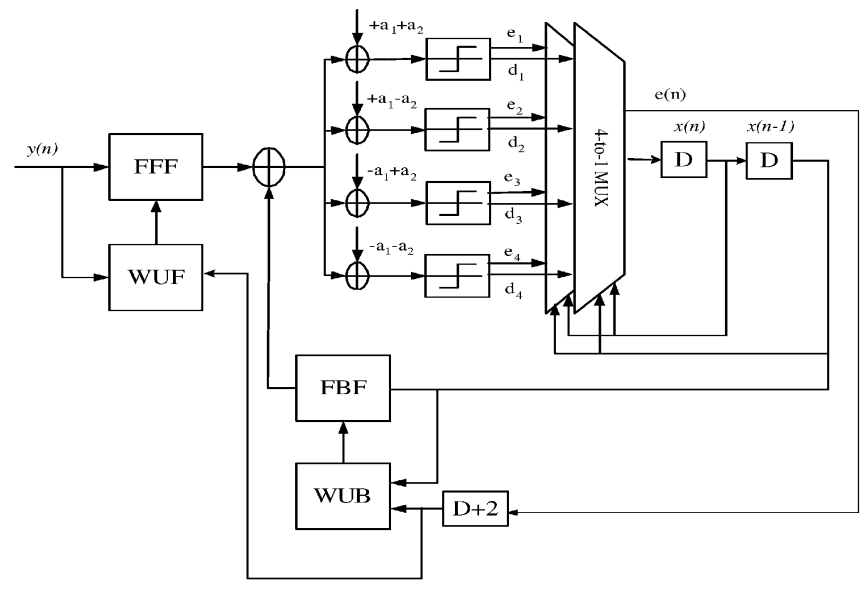

Fig. 13. Two-stage look-ahead architecture of the pipelining DFE [13]. WUF and WUB denote weight updating blocks for FFF and FBF, respectively.

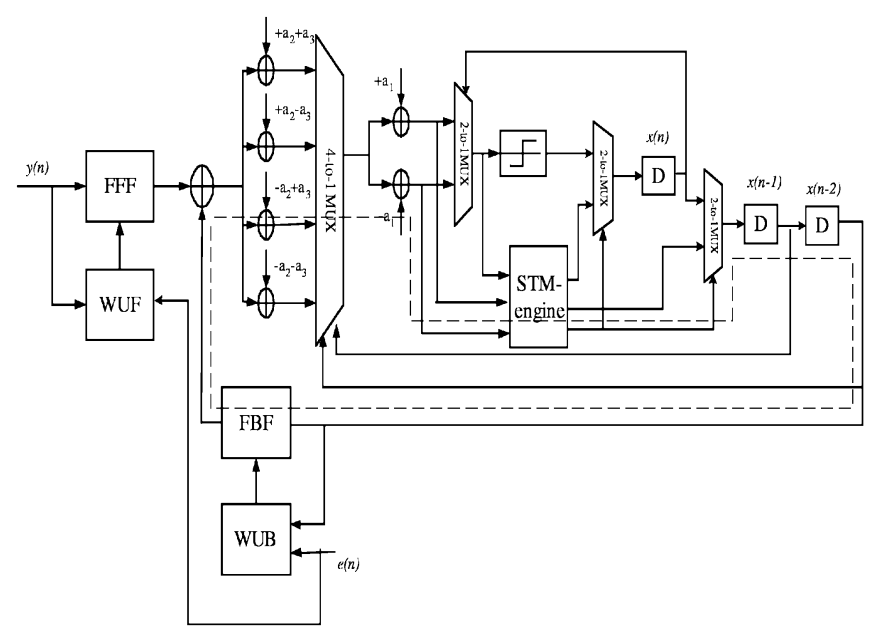

Fig. 14. Overall system architecture of the two-layer PSTM-DFE with two-stage look-ahead. The block WUF and WUB denote weight updating blocks for FFF and FBF, respectively.

therefore, it will be useless for the look-ahead of $a_{1}$. For the two-stage look-ahead, we need to expand $a_{2}$ and $a_{3}$ and not $a_{1}$ and $a_{2}$.

The overall system diagram of the STM-DFE is depicted in Fig. 14. The critical loop of the STM-DFE is lined in dashes. 
TABLE II

SuMmary OF HARDWARE COMPLEXITY BETWEen CONVENTIONAL PIPELINE DFE AND PSTM-DFE FOR N-STAGE LOOK-AHEAD. W (BITS) IS DEPENDENT ON the Target SNR Performance. In This Table, We Do Not Take the FFF, WUF, FBF, And WUB Into Account

\begin{tabular}{c|c|c|c|c|c}
\hline Equalizer type & $\begin{array}{c}\text { Number } \\
\text { of Adders }\end{array}$ & $\begin{array}{c}\text { Number of } \\
\text { Threshold } \\
\text { Detectors }\end{array}$ & $\begin{array}{c}\text { Number } \\
\text { of Squares }\end{array}$ & Number of MUX & Speed Up \\
\hline $\begin{array}{c}\text { Conventional } \\
\text { pipeline DFE }\end{array}$ & $1+2^{N}$ & 0 & 0 & One $2^{N}$-to-1 (1 bit) & $M=(N+1)$ \\
\hline PSTM-DFE & $6+2^{N}$ & 1 & 3 & One 2 - to-1 (W bits $)$ & $M=(N+1)$ \\
\hline
\end{tabular}

Table II summarizes the total overhead of the PSTM-DFE and the conventional DFE.

\section{CONCLUSION}

This work proposes a novel and effective STM-DFE algorithm to enhance the DFE performance. At the algorithm level, the threshold of the reliable/unreliable region is analyzed in closed form. At the architecture level, the STM-DFE is very suitable for VLSI design. Its hardware cost is very similar to that of the simple DFE. In summary, the proposed STM algorithm can outperform the conventional DFE low hardware overhead. The PSTM-DFE is also suitable for high-speed communications and storage applications.

\section{APPENDIX \\ DERIVATION OF BOUNDARY VALUES}

We first derive the value of the boundary $A$. Based on (7), $\bar{r}_{n+1}$ must satisfy

$$
\begin{aligned}
\operatorname{Pr}\left(\bar{r}_{n+1} \mid+1,+1\right) & \times \operatorname{Pr}\left(r_{n} \mid+1\right) \\
& =\operatorname{Pr}\left(\bar{r}_{n+1} \mid+1,-1\right) \times \operatorname{Pr}\left(r_{n} \mid-1\right) .
\end{aligned}
$$

Applying Assumption A2), (23) can be reformulated as

$$
\begin{aligned}
& \exp \left(\frac{\left(\bar{r}_{n+1}-1-a_{1}\right)^{2}}{-2 \varepsilon_{d}^{2}}+\frac{\left(r_{n}-1\right)^{2}}{-2 \varepsilon_{d}^{2}}\right) \\
& =\exp \left(\frac{\left(\bar{r}_{n+1}-1+a_{1}\right)^{2}}{-2 \varepsilon_{d}^{2}}+\frac{\left(r_{n}+1\right)^{2}}{-2 \varepsilon_{d}^{2}}\right) .
\end{aligned}
$$

By simplifying (24), we have

$$
\bar{r}_{n+1}=1-r_{n} / a_{1} .
$$

Therefore, the boundary $A$ is equal to $1-r_{n} / a_{1}$. Boundaries $B$ and $C$ can be derived in the same way.

\section{REFERENCES}

[1] P. Monsen, "Adaptive equalization of the slow fading channel," IEEE Trans. Commun., vol. COM-22, no. 8, pp. 1064-1075, Aug. 1974.

[2] A. P. Clark and M. Clayden, "Pseudo-binary Viterbi detector," Proc. Inst. Elect. Eng. F, vol. 131, pp. 208-218, Apr. 1984.

[3] M. V. Eyuboglu and S. U. H. Qureshi, "Reduced-state sequence estimation with set partitioning and decision feedback," IEEE Trans. Commun., vol. 36, no. 1, pp. 13-20, Jan. 1988.

[4] R. P. Lippmann, "An introduction to computing with neural nets," in IEEE Acoust., Speech, Signal Process. Mag., vol. 4, Apr. 1987.
[5] G. Mathew, Y. X. Lee, and V. Y. Krachkovsky, "A novel threshold technique for minimizing error propagation in MDFE read channel," in Proc. IEEE GLOBECOM Conf., Sydney, Australis, Nov. 1998, pp. 2898-2903.

[6] F. Zhao, G. Mathew, and B. Farhang-Boroujeny, "Techniques for minimizing error propagation in decision feedback detectors for recording channels," IEEE Trans. Magn., vol. 37, no. 1, pp. 592-602, Jan. 2001.

[7] B. E. Moision, P. Seigel, and E. Soljanin, "Error event characterization and coding for the equalized lorentzian channel," in Proc. ISIT, Cambridge, MA, USA, Aug. 16-21, 1998.

[8] J. G. Proakis, Digital Communications, Fourth ed. New York: McGraw-Hill, 2001

[9] K. K. Parhi, "Pipelining in algorithm with quantizer loops," IEEE Trans. Circ. Syst., vol. 38, no. 7, pp. 745-754, Jul. 1991.

[10] N. R. Shanbhag and K. K. Parhi, "Pipelined adaptive ADFE architectures using relaxed look-ahead," IEEE Trans. Signal Process., vol. 43, no. 6, pp. 1368-1385, Jun. 1995.

[11] K. K. Parhi, VLSI Digital Signal Processing System. New York: Wiley, 1999.

[12] M. D. Yang, A.-Y. Wu, and J. T. Lai, "Fast convergent pipelined adaptive DFE architecture using post-cursor processing filter technique," IEEE Trans. Circuits Syst. II: Analog Digit. Signal Process., vol. 51, no. 2, pp. 57-60, Feb. 2004.

[13] — - "High-performance VLSI architecture of adaptive decision feedback equalizer based on predictive parallel branch slicer (PPBS) scheme," IEEE Trans. VLSI Syst., vol. 12, no. 2, pp. 218-226, Feb. 2004.

[14] E. T. Fiez, K. Winter, and T. Fisher, "Design of a new squaring function for the Viterbi algorithm," IEEE J. Solid-State Circuits, vol. 29, no. 9, pp. 1102-1107, Sep. 1994.

[15] A. A. Hiasat and A. S. Abdel-Aty-Zohdy, "Combinational logic approach for implementing an improved approximate squaring function," IEEE J. Solid-State Circuits, vol. 34, no. 2, pp. 236-240, Feb. 1999.

[16] E. F. Haratsch and K. Azadet, "High-speed reduced-state sequence estimation," in Proc. IEEE Int. Symp. Circuits Syst., vol. 3, Geneva, Switzerland, May 2000, pp. 387-390.

[17] C. Caraiscos and B. Liu, "A roundoff error analysis of the LMS adaptive algorithm," IEEE Trans. Acoust., Speech, Signal Process., vol. ASSP-32, no. 1, pp. 34-41, Feb. 1984.

[18] G. Long, F. Ling, and J. G. Proakis, "The LMS algorithm with delayed coefficient adaptation," IEEE Trans. Signal Process., vol. 37, pp. 1397-1405, Sep. 1989.

[19] M. V. Eyuboglu and S. U. Qureshi, "Reduced-state sequence estimation for coded modulation on intersymbol interference channels," IEEE J. Sel. Areas Commun., vol. 7, no. 8, pp. 989-995, Aug. 1989.

[20] H. N. Kim, S. I. Park, and S. W. Km, "Performance analysis of error propagation effects in the DFE for ATSC DTV receivers," IEEE Trans. Broadcast., vol. 49, no. 3, pp. 249-257, Sept. 2003.

[21] V. Y. Krachkovsky et al., "Error propagation evaluation for RLL-constrained DFE read channels," IEEE Trans. Magn., vol. 34, no. 1, pp. 147-152, Jan. 1998.

[22] J. E. Smee and N. C. Beaulieu, "Error-rate evaluation of linear equalization and decision feedback equalization with error propagation," IEEE Trans. Commun., vol. 46, no. 5, pp. 656-665, May 1998.

[23] M. Chiani, "Introducing erasures in decision-feedback equalization to reduce error propagation," IEEE Trans. Commun., vol. 45, no. 7, pp. 757-760, Jul. 1997.

[24] M. Tarrab and A. Feuer, "Convergence and performance analysis of the normalized LMS algorithm with uncorrelated Gaussian data," IEEE. Trans. Acoust., Speech, Signal Process., vol. 34, pp. 680-691, Jul. 1988. 
[25] S. Koike, "Analysis of adaptive filters using normalized singed regressor LMS algorithm," IEEE Trans. Signal Process., vol. 47, no. 8 , pp. 2710-2723, Oct. 1999.

[26] E. Eweda, "Analysis and design of a signed regressor LMS algorithm for stationary and nonstationary adaptive filtering with correlated Gaussian data," IEEE Trans. Circuits Syst., vol. 37, no. 11, pp. 1367-1374, Nov. 1990.

[27] D. I. Kim and P. D. Wilde, "Performance analysis of singed self-orthogonalizing adaptive lattice filter," IEEE Trans. Circ. Syst. II: Analog Digit. Signal Process., vol. 47, no. 9, pp. 1227-1237, Oct. 2000.

[28] B. Farhang-Boroujeny, "Fast LMS/Newton algorithms based on autoregressive modeling and their application to acoustic echo cancellation," IEEE Trans. Signal Process., vol. 45, no. 8, pp. 1987-1997, Aug. 1997.

[29] S. Haykin, Adaptive Filter Theory, Second ed. Englewood Cliffs, NJ: Prentice-Hall, 1991.

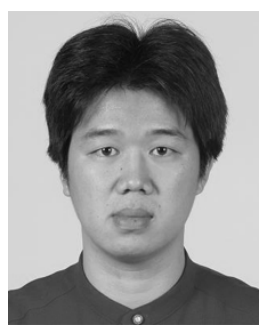

Chih-Hsiu Lin received the B.S. degree in mechanical engineering in 1998 from National Taiwan University, Taipei, Taiwan, R.O.C., where he is currently working toward Ph.D. degree with the Graduate Institute of Electronics Engineering.

His research interests include the VLSI implementation of communication systems and multiuser detections.

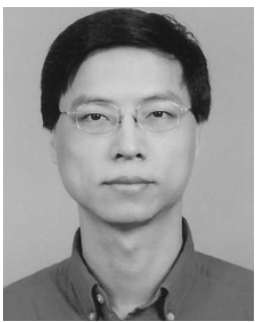

An-Yeu (Andy) Wu received the B.S. degree from National Taiwan University, Taipei, Taiwan, R.O.C., in 1987 and the M.S. and Ph.D. degrees from the University of Maryland, College Park, in 1992 and 1995, respectively, all in electrical engineering.

From August 1995 to July 1996, he was a Member of Technical Staff at AT\&T Bell Laboratories, Murray Hill, NJ, working on high-speed transmission IC designs. From 1996 to July 2000, he was with the Electrical Engineering Department, National Central University, Chung-Li, Taiwan. He is currently an Associate Professor with the Graduate Institute of Electronics Engineering and Department of Electrical Engineering, National Taiwan University. His research interests include low-power/high-performance VLSI architectures for DSP and communication applications, adaptive/multirate signal processing, and reconfigurable broadband access systems and architectures.

Dr. Wu served as an Associate Editor for the EURASIP Journal of Applied Signal Processing from 2001 to 2004. He became the Associate Editor of the IEEE TRANSACTIONS ON VERY LARGE SCALE INTEGRATION SYSTEMS since July 2003. He has served on the technical program committees of IEEE International Conferences such as ICIP, SiPS, AP-ASIC, ISCAS, and SOC. He received the Research Award from National Science Council for four times during 1997 and 2000. He also received the Macronix International Corporation (MXIC) Young Chair Professor Award in 2003. 